\title{
Exploración del conocimiento y percepción de los es- tudiantes de Medicina del último año de la Facultad de Medicina de la Universidad Nacional del Nordeste sobre los medicamentos genéricos en los meses agosto a octubre del año 2020
}

\begin{abstract}
Exploration of the knowledge and perception of the last year medical students of the Faculty of Medicine of the Northeast National University on generic drugs in the months of August to October of the year 2020

Ignacio Pinedo ${ }^{1}$; Mariela Lucía Scheiber ${ }^{1}$; Facundo David Vargas Capará1 ${ }^{\prime}$ Cecilia Villalba ${ }^{1}$; Rosana Gerometta ${ }^{2}$
\end{abstract}

\section{RESUMEN}

Introducción: Según la Organización Mundial de la Salud, un medicamento genérico es "un producto farmacéutico, generalmente destinado a ser intercambiable con un producto innovador, que se fabrica sin una licencia de la empresa innovadora y se comercializa después de la fecha de vencimiento de la patente u otros derechos exclusivos". Como futuros médicos que prescribirán, tienen un papel muy importante en la implementación y promoción de los medicamentos genéricos. El objetivo de este trabajo es determinar los conocimientos sobre medicamentos genéricos en estudiantes de Medicina del último año de la Facultad de Medicina de la Universidad Nacional del Nordeste. Metodología: Estudio descriptivo, transversal con los estudiantes que cursan el último año de la carrera. Para obtener los datos se elaboró una encuesta en Google Forms que enviada a los estudiantes. Posteriormente se creó una base de datos en Excel y se analizó con Epi Info V7. Resultados: El 92.2\% consideró que las diversas marcas comerciales del mismo fármaco no modifican la acción del mismo. Casi la totalidad de los estudiantes se encuentran seguros de poder aconsejar en un futuro fármacos por su nombre genérico. La totalidad de los encuestados indican que tanto ellos como los pacientes que pueden observar en sus prácticas académicas solicitan mayor información sobre el margen de seguridad de los medicamentos genéricos. Conclusión: Como futuros médicos, se considera que los estudiantes deben tener un alto nivel de conocimiento sobre los medicamentos genéricos para prescribir correctamente y aconsejar e informar adecuadamente a sus pacientes, familiares y/o amigos.

Palabras clave: Medicamentos; Estudiantes; Medicina; Conocimientos.

Fecha de recepción: octubre 2020; fecha de aceptación: noviembre 2020

${ }^{1}$ Universidad Nacional del Nordeste de Argentina, Carrera de Medicina. Argentina

${ }^{2}$ Universidad de Buenos Aires. Facultad de Medicina. Argentina

Autor de correspondencia: Ignacio Pinedo. Email: ignacio20.ip@gmail.com 


\section{ABSTRACT}

Introduction: According to the World Health Organization, a generic drug is "a pharmaceutical product, generally intended to be interchangeable with an innovative product, that is manufactured without a license from the innovative company and is marketed after the expiration date of the patent or other exclusive rights". As future prescribing physicians, they play a very important role in the implementation and promotion of generic drugs. The objective of this work is to determine the knowledge about generic drugs in medical students of the last year of the Faculty of Medicine of the National University of the Northeast. Methodology: Descriptive, cross-sectional study with students who are in the last year of the degree. To obtain the data, a survey was prepared in Google Forms that was sent to the students. Subsequently, a database was created in Excel and analyzed with Epi Info V7. Results: $92.2 \%$ considered that the various commercial brands of the same drug do not modify its action. Almost all of the students are sure of being able to advise drugs by their generic name in the future. All the respondents indicate that both they and the patients they can observe in their academic practices request more information about the safety margin of generic drugs. Conclusion: As future doctors, it is considered that students should have a high level of knowledge about generic drugs to correctly prescribe and properly advise and inform their patients, family members and / or friends.

Keywords: Medications; Students; Medicine; Knowledge. 


\section{INTRODUCCIÓN}

La Organización Mundial de la Salud (OMS) define a los medicamentos genéricos (MG) como "un producto farmacéutico, generalmente destinado a ser intercambiable con un producto innovador, que se fabrica sin una licencia de la empresa innovadora y se comercializa después de la fecha de vencimiento de la patente $u$ otros derechos exclusivos" (1). Un MG funciona de la misma manera y proporciona el mismo beneficio clínico que el mismo medicamento, en la misma dosis y proporción que un medicamento de marca comercial (MC). Esta norma se aplica a todos los MG aprobados por la Agencia de Administración de Medicamentos y Alimentos (FDA). Un MG es lo mismo que un medicamento de marca en dosis, seguridad, eficacia, concentración, estabilidad y calidad, así como en la forma en que se toma y debe usarse. Los MG o fármacos genéricos (FG) solo se hacen disponibles después de una examinación rigurosa por parte de la FDA y luego de un período de tiempo determinado de exclusividad de la MC. Esto se debe a que los medicamentos nuevos, como otros productos, generalmente están protegidos por patentes que prohíben que otros fabriquen y vendan copias del mismo medicamento. La patente tiene como objetivo principal proteger la inversión de la compañía en el desarrollo del medicamento al otorgar a la empresa el derecho exclusivo de vender el medicamento mientras la misma se encuentre vigente. Debido a la duración de llevar un nuevo medicamento al mercado, este período de exclusividad permite a las compañías farmacéuticas recuperar los costos asociados con la comercialización de un nuevo medicamento. La FDA también otorga ciertos períodos de exclusividad de comercialización a MC que pueden prohibir la aprobación de $\mathrm{MG}$ por lo cual, una vez que estas patentes y exclusividades de comercialización caducan (o si las patentes son impugnadas con éxito por la compañía de MG), se puede aprobar el MC solicitante. Los MG también tienden a costar menos que sus equivalentes de marca debido a que los fabricantes solicitantes no tienen que repetir los estudios en animales y humanos que fueron exigidos a los de una determinada MC para demostrar su seguridad y eficacia. La accesibilidad se ve a menudo obstaculizada, entre otros, por los altos precios de estos medicamentos (2). Un tercio de la población del mundo en desarrollo no tiene acceso a medicamentos (3). Como estrategia para aumentar el acceso, la mayoría de los países desarrollados y en proceso de desarrollo han instituido medidas para reducir gastos en medicamentos. Uno de estos enfoques implica promover el uso de $\mathrm{MG}$ entre los proveedores de atención médica (4).

Por esto, la solicitud se la denomina "nueva solicitud de medicamento abreviada", que, junto con la competencia entre el MC y múltiples MG, es en gran parte la razón por la cual los MG cuestan mucho menos (5) y permite también, ser una medida rentable reduciendo gastos de atención médica en productos farmacéuticos, generando ahorros sustanciales tanto para la población como para el Estado (6).

Como futuros profesionales que prescribirán y serán responsables de las políticas sanitarias, los médicos tienen un papel muy importante en la implementación y promoción de los MG (7).

Por tales motivos surgió en el equipo de investigación, la inquietud de explorar el conocimiento y las actitudes que tienen los alumnos del último año de la carrera de Medicina sobre los medicamentos genéricos.

\section{Objetivos: \\ General:}

- Determinar los conocimientos 
sobre medicamentos genéricos en estudiantes de Medicina del último año de la Facultad de Medicina de la Universidad Nacional del Nordeste.

\section{Específicos:}

- Describir las características sociodemográficas de los estudiantes involucrados.

\section{MÉTODO}

Se realizó un estudio descriptivo, transversal con los estudiantes que cursan el último año de la carrera de medicina de la Universidad Nacional del Nordeste.

\section{Criterios de Inclusión:}

Estudiantes regulares de la carrera de Medicina de la Facultad de Medicina Universidad Nacional del Nordeste pertenecientes al último año de cursado ( $6^{\circ}$ año).

\section{Criterios de Exclusión:}

Estudiantes libres.

Como herramienta de recolección de datos se utilizó un cuestionario prediseñado en la plataforma Google Forms que los estudiantes pudieron completarlo de forma online con carácter anónimo durante los meses agosto - octubre de 2019 con previa validación del mismo mediante una prueba piloto realizada a 10 estudiantes pertenecientes a la muestra, para poder va-
- Identificar los conocimientos acerca de los medicamentos genéricos en el grupo seleccionado. lorar la comprensión y pertinencia de las preguntas realizadas. El cuestionario fue enviado mediante la red social Whatsapp y los resultados del mismo fueron volcados a una planilla Excel 2016 para su posterior análisis estadístico mediante el software Epi Info V7. Las respuestas se observaron mediante la escala Likert normal de 5 puntos (Totalmente de acuerdo, de acuerdo, neutral, en desacuerdo y totalmente en desacuerdo).

El cuestionario estaba constituido en una primera sección con preguntas sobre aspectos sociodemográficos. La siguiente sección, estuvo abocada al nivel de conocimiento sobre medicamentos genéricos. Los datos fueron almacenados automáticamente en una planilla de cálculo Excel 2016 y analizados mediante técnicas estadísticas con el programa Epi Info V7.

\section{RESULTADOS}

Las encuestas fueron enviadas a 147 personas de los cuales fueron respondidas en su totalidad por $64(n=64)$. La edad media fue de 25,1 años con un $\mathrm{DE} \pm 1,5$ años de edad. El $84.4 \%$ solamente estudian y el $15.6 \%$ restante estudian y trabajan. El $51.6 \%$ fueron mujeres. $92.2 \%$ de los estudiantes se encontraban solteros y el restante en pareja. $48.4 \%$ viven solos, $35.9 \%$ con su familia, $7.8 \%$ con su pareja y el $7.8 \%$ restante con amigos o compañeros.

Al consultar respecto al momento de comprar o consumir $62.5 \%$ prefieren un
MG antes que un MC, 31.3\% depende de la situación o medicamento a necesitar y $6.3 \%$ elijen un MC conocido. El 62.5\% cuando son consultados por amigos y/o familiares sobre alguna preferencia de un medicamento les recomienda comprar un MG frente al 37.5\% que aconsejan elegir alguna MC determinada.

E1 92.2\% de los universitarios encuestados considero que las diversas MC del mismo fármaco no varían en efectividad y solamente un $7.8 \%$ mencionó que la efectividad de un tratamiento farmacológico 
depende mucho de la MC y el laboratorio que lo produce.

Como podemos ver reflejado en la tabla $\mathrm{N}^{\circ} 1$, casi la totalidad de los estudiantes se encuentran seguros de poder aconsejar en un futuro, fármacos por su nombre genérico en el tratamiento de sus pacientes. Más de la mitad de los encuestados piensa que las publicidades influyen en la compra de un medicamento al igual que consideran que necesitan información adecuada y suficiente sobre la seguridad de un fármaco determinado. El 98.5\% discurre que los farmacéuticos actúan como consejeros al momento de recomendar un medicamento y el $96.9 \%$ que el paciente debe recibir información suficiente para elegir qué tipo medicamentos consume.

Tabla 2: Porcentaje de estudiantes de medicina del último año de la Facultad de Medicina de la Universidad Nacional del Nordeste sobre la percepción de los medicamentos genéricos en los meses agosto a octubre del año 2020. $(n=64)$

\begin{tabular}{|c|c|c|c|c|c|}
\hline & $\begin{array}{l}\text { Totalmente de } \\
\text { acuerdo. }\end{array}$ & De acuerdo & Neutral. & En desacuerdo. & $\begin{array}{l}\text { Muy en des- } \\
\text { acuerdo }\end{array}$ \\
\hline $\begin{array}{l}\text { Por el conocimiento que } \\
\text { tengo, estoy seguro de } \\
\text { poder dar consejos en el } \\
\text { futuro por el nombre del } \\
\text { medicamento genérico } \\
\text { en lugar del nombre de } \\
\text { marca. }\end{array}$ & 50 & 43,8 & 4,7 & 1,5 & - \\
\hline $\begin{array}{l}\text { Creo que la publicidad de } \\
\text { las compañias farmacéu- } \\
\text { ticas influirá en el uso de } \\
\text { medicamentos de marca. }\end{array}$ & 34,4 & 64,1 & 1,5 & - & - \\
\hline $\begin{array}{l}\text { Necesito más informa- } \\
\text { ción sobre las cuestiones } \\
\text { relativas a la seguridad y } \\
\text { eficacia de los medica- } \\
\text { mentos genéricos. }\end{array}$ & 40,6 & 59,4 & - & - & - \\
\hline $\begin{array}{l}\text { Creo que los farma- } \\
\text { céuticos son uno de los } \\
\text { profesionales sanitarios } \\
\text { más importantes para dar } \\
\text { consejos sobre medica- } \\
\text { mentos genéricos. }\end{array}$ & 35,9 & 62,6 & 1,5 & - & - \\
\hline $\begin{array}{l}\text { Creo que el paciente } \\
\text { debe recibir suficiente } \\
\text { información sobre los } \\
\text { medicamentos genéricos } \\
\text { para asegurarse de que } \\
\text { realmente comprenda los } \\
\text { medicamentos que toma. }\end{array}$ & 67,2 & 29,7 & 3,1 & - & - \\
\hline
\end{tabular}

Al consultar sobre los conocimientos respecto a los $\mathrm{MG}$, como detalla la tabla $\mathrm{N}^{\circ} 2$ a continuación, podemos observar que el $98.5 \%$ de los estudiantes pertenecientes a la muestra consideran que los MG son bioequivalente respecto a diferente $\mathrm{MC}$. El 97\% opina que los MG deben tener la misma forma de proporción que la $\mathrm{MC}$, el $89 \%$ menciona que también debe contener la misma dosis y el $81 \%$ que debe ser el 
mismo envase. E1 37.5\% considera que los tos secundarios. El 62.5\% refiere que los MG no tienen menor calidad que los MC. MG son menos costosos y más accesibles El 68.8\% menciona que los medicamentos para la población. Y, el 82.8\% confía en de marca deben cumplir con estándares de poder prescribir y dispensar según el nomseguridad más altos que los genéricos. Sin bre genérico de un medicamento cuando embargo, el $37.5 \%$ de los universitarios sus pacientes lo requieran. considera que los MG producen más efec-

Tabla 2: Porcentaje de estudiantes de medicina del último año de la Facultad de Medicina de la Universidad Nacional del Nordeste sobre conocimientos de medicamentos genéricos en los meses agosto a octubre del año 2020. ( $n=64)$

\begin{tabular}{|c|c|c|c|c|c|}
\hline & $\begin{array}{c}\text { Totalmente de } \\
\text { acuerdo. }\end{array}$ & De acuerdo & Neutral. & En desacuerdo. & $\begin{array}{l}\text { Muy en des- } \\
\text { acuerdo }\end{array}$ \\
\hline $\begin{array}{l}\text { Un medicamento genérico } \\
\text { es bioequivalente a un } \\
\text { medicamento de marca. }\end{array}$ & 34,4 & 64,1 & 1,5 & - & - \\
\hline $\begin{array}{l}\text { Un medicamento genéri- } \\
\text { co debe estar en la misma } \\
\text { forma de dosificación que } \\
\text { el medicamento de marca. }\end{array}$ & 18,8 & 78,2 & 1,5 & 1,5 & - \\
\hline $\begin{array}{l}\text { Un medicamento } \\
\text { genérico debe contener la } \\
\text { misma dosis que los medi- } \\
\text { camentos de marca. }\end{array}$ & 15,6 & 73,4 & 7,8 & 2,3 & - \\
\hline $\begin{array}{l}\text { Un medicamento genérico } \\
\text { debe estar en la misma } \\
\text { forma de dosificación } \\
\text { como ser tableta, cápsula u } \\
\text { otros que el medicamento } \\
\text { de marca. }\end{array}$ & 14,1 & 67,2 & 12,5 & 6,2 & - \\
\hline $\begin{array}{c}\text { Los medicamentos genéri- } \\
\text { cos son de calidad inferior } \\
\text { a los medicamentos de } \\
\text { marca. }\end{array}$ & 4,7 & 20,3 & 37,5 & 25 & 12,5 \\
\hline $\begin{array}{l}\text { Los medicamentos genéri- } \\
\text { cos producen más efectos } \\
\text { secundarios que los medi- } \\
\text { camentos de marca. }\end{array}$ & - & 37.5 & 34.4 & 20.3 & 7.8 \\
\hline $\begin{array}{l}\text { Los medicamentos gené- } \\
\text { ricos son menos costosos } \\
\text { que los de marca. }\end{array}$ & 10,9 & 51,6 & 23,4 & 14,1 & - \\
\hline $\begin{array}{l}\text { Los medicamentos de } \\
\text { marca deben cumplir con } \\
\text { estándares de seguridad } \\
\text { más altos que los medica- } \\
\text { mentos genéricos. }\end{array}$ & 14,1 & 54,7 & 18,8 & 10,9 & 1,5 \\
\hline $\begin{array}{l}\text { Por el conocimiento que } \\
\text { tengo, confío en dispensar } \\
\text { en el futuro por nombre } \\
\text { de medicamento genérico } \\
\text { en lugar de por nombre de } \\
\text { marca. }\end{array}$ & 48,4 & 34,4 & 10,9 & 6,3 & - \\
\hline
\end{tabular}




\section{DISCUSIÓN}

Cubrir las necesidades financieras dentro de nuestro sistema sanitario, tradicionalmente ha sido una tarea fundamental. Es así que más aun, hoy día, se hace necesario que poder ejecutivo, administraciones centrales, agentes sanitarios, instituciones y ciudadanos, sumemos esfuerzos; y que de manera coherente y coordinada trabajemos en la misma dirección: preservar el sistema público de salud concediéndole los recursos suficientes para mantener los estándares de calidad de la salud pública, como uno de los mayores logros sociales alcanzados en las últimas décadas.

El acceso al medicamento ocupa un lugar preferencial en esta rutina económica y la administración de fármacos eficaces a un menor precio, mejora los índices de administración en el sistema de salud. Por ello, el conocimiento de los estudiantes de Medicina sobre los MG, fortalece la gestión futura.

Al consultar a nuestros estudiantes respecto al momento de comprar o consumir fármacos, un $62.5 \%$ prefieren un MG antes que un $\mathrm{MC}, 31.3 \%$ depende de la situación o medicamento que necesiten $\mathrm{y}$ $6.3 \%$ elijen un MC conocido. El 62.5\% cuando son consultados por amigos y/o familiares sobre alguna preferencia de un medicamento les recomienda comprar un $\mathrm{MG}$ frente al 37.5\% que aconsejan elegir alguna MC determinada.

E1 92.2\% de los universitarios encuestados consideró que las diversas MC del mismo fármaco son solo un negocio de los diferentes laboratorios y que es independiente de la efectividad de un tratamiento y solamente un $7.8 \%$ que la efectividad de un tratamiento farmacológico depende mucho de la MC y el laboratorio que lo produce.

Según James et al. (2018) el 85.7\% de la población estudiada confía en tener conocimiento suficientes y adecuados para aconsejar en un futuro sobre un MG en lugar del comercial, un valor similar fue obtenido en nuestro estudio con un 93.8\% 4 .

En un trabajo publicado por Gyawali et al. (2016), menciona que un $81.8 \%$ de los encuestados considera que la publicidad de parte de las diferentes compañías farmacéuticas influye en el uso y compra de determinada $\mathrm{MC}$, frente a un 98.5\% encontrado en nuestro estudio 7 .

Es de destacar el interés de los encuestados por más datos sobre MG. Según nuestros hallazgos, la totalidad de los estudiantes $(100 \%)$ solicita mayor información respecto a seguridad y eficacia de los MG. Lo que coincide con las afirmaciones de otros autores que también trabajaron con estudiantes universitarios de ciencias de la salud, como por ejemplo James et al. (2018) que encontraron un $88.6 \%$ de estudiantes que requerían mayor información y Gyawali et al. (2016) en un 92.9\% 4, 7.

En la cadena de dispensación, el profesional farmacéutico juega un rol muy importante, valorado por nuestros estudiantes en un $98.5 \%$, coincidente con el estudio de James et al. (2018) que menciona un $97.6 \%$ de la muestra de acuerdo con el papel del farmacéutico 4.

Según Gyawali et al. (2016), el 86.5\% de la población encuestada considera que el paciente debe recibir suficiente información sobre los MG para asegurarse de que realmente comprenda sobre los medicamentos que toma, valor similar al obtenido por nosotros, un $96.9 \%$. Se considera que educar al paciente sobre su prescripción, calidad, cantidad, momento de la administración, posibles efectos adversos, contribuye a la adherencia al tratamiento 7.

Surgieron diferencias en los porcentajes encontrados sobre consideraciones de bioequivalencia. Según James et al. (2018) los estudiantes de Medicina en un 
$31 \%$ opinaban que un $\mathrm{MG}$ es bioequivalente a su homólogo de marca y Gyawali et al. (2016) encontraron ese dato en un $64.1 \%$ de los estudiantes incluidos. Sin embargo, nuestros resultados arrojaron que un $98.5 \%$ de la muestra considera bioequivalente al MG y MC 4,7.

Con respecto a la dosis del MG, según datos de James et al. (2018) un 67.7\% respondió que debe tener la misma dosis el MG en relación al MC con similares porcentajes al estudio de Gyawali et al. (2016) 72.6\%. En nuestro caso, un $89 \%$ de los estudiantes consideran que deberían tener la misma dosis. Tal vez esta apreciación fundamente el alto porcentaje de estudiantes que en nuestro estudio

\section{CONCLUSIONES}

Se pudo observar un gran porcentaje de estudiantes que prefieren comprar o consumir MG antes que las MC. Además, se apreció un alto nivel conocimiento sobre los medicamentes en los estudiantes. Consideramos que esto es muy importante, ya que la mayor parte son consultados por familiares y/o amigos y aconsejan sobre el uso de determinados MG o MC.

Por otro lado, también discurrimos en que es muy importante que los estudiantes tengan un alto nivel de conocimiento so- afirmó que MG y MC son bioequivalente, evidenciando la falta de diferenciación del concepto de bioequivalencia 4,7.

Al indagar sobre la calidad de los MG, nuestros estudiantes en un $75 \%$ afirmaron que no veían diferencias con los MC cifras similares a las halladas por James et al. (2018) con 81\%.

Según estos autores un $54,8 \%$ estaban en contra de la afirmación de que un $\mathrm{MG}$ tiene más efectos secundarios, similares datos encontrados por Gyawali et al. (2016) el 60.3\%. Se observa una diferencia en nuestro estudio donde el $28.1 \%$ de nuestros estudiantes considera que podrían generar mayores efectos adversos 4,7.

bre los medicamentos para poder informar correctamente a las personas de su entorno o futuros pacientes. Además, muchas veces los consumidores al no tener la información adecuada, desconocen las consecuencias que podría traer aparejada el consumo de determinados medicamentos.

Se sugiere continuar con la línea de investigación para aumentar el conocimiento sobre los estudiantes y de ser necesario, evaluar otras alternativas de aprendizaje.

\section{REFERENCIAS}

1. Comité de Expertos de la OMS en Especificaciones para las Preparaciones Farmacéuticas: 32a informe, Ginebra, 10-15 de diciembre de 1990 [Internet]. Ginebra: Organización Mundial de la Salud; [citado 22 de octubre de 2020]. Disponible en: https://apps.who.int/iris/handle/10665/41379

2. Hogerzeil HV, Mirza Z. Situación mundial de los medicamentos 2011: Acce- so a medicamentos esenciales como parte del derecho a la salud. Organización Mundial de la Salud. Ginebra, Suiza; 2011 p. 680-9.

3. Lu Y, Hernández $\mathrm{P}$, Abegunde $\mathrm{D}$, Edejer T. La situación mundial de los medicamentos 2011. Gastos en medicamentos. Organización Mundial de la Salud. Ginebra, Suiza; 2011. 
4. James PB, Bah AJ, Margao EK, sali MA, Sharrad AK, Shafie AA, Babar Hanson C, Kabba JA, Jamshed SQ. Explo- Z-U-D. Understanding and perceptions ring the Knowledge and Perception of Ge- of final-year Doctor of Pharmacy students neric Medicines among Final Year Under- about generic medicines in Karachi, Pakisgraduate Medical, Pharmacy, and Nursing tan: a quantitative insight. Adv Med Educ Students in Sierra Leone: A Comparative Pract. 15 de mayo de 2015;6:359-66. Cross-Sectional Approach. Pharmacy (Basel). 4 de enero de 2018;6(1):3.

5. Food \& Drug Administration. Medicamentos Genéricos: Preguntas y Respuestas [Internet]. [citado 25 de octubre de 2020]. Disponible en: https://www.fda. gov/media/112590/download

6. Jamshed SQ, Ibrahim MIM, Has-

7. Gyawali S, Hassali MA, Saha A. A survey exploring the knowledge and perceptions of senior medical students in Nepal toward generic medicines. SAGE Open Med [Internet]. 4 de agosto de 2016 [citado 27 de octubre de 2020];4. Disponible en: https://www.ncbi.nlm.nih.gov/ pmc/articles/PMC4976792/ 